$17^{\text {th }}$ International Congress of Metrology, 02002 (2015)

DOI: $10.1051 /$ metrology / 201502002

C) Owned by the authors, published by EDP Sciences, 2015

\title{
L’approche Monte Carlo à plusieurs niveaux pour évaluer le processus au plus proche de la physique
}

\author{
François Hennebelle ${ }^{1, a}$, Thierry Coorevits ${ }^{2}$, Renald Vincent ${ }^{3}$ \\ ${ }^{1}$ LE2I UMR6306, CNRS, Arts et Métiers, Univ. Bourgogne Franche-Comté, F-21000 Dijon, France \\ ${ }^{2}$ Laboratoire MSMP, Arts et Métiers Paris Tech - 8 Boulevard Louis XIV, 59046 LILLE Cedex \\ ${ }^{3}$ Centre Technique des Industries Mécaniques (CETIM) - 52, avenue Félix-Louat - BP 80067 - 60304 Senlis Cedex2
}

\begin{abstract}
Monte Carlo method have been introduced in metrology in the 1990's and integrated in the GUM (Supplement 1) in 2008. This method is more and more used. Typically the users of this method realize a complete simulation in one step, like the GUM, one step for one model. This is unfortunate, the simulation loses its physical sense. The study aim is to present a multi-level Monte Carlo approach which allows being near of the reality of the measurement process. Two applications are developed: evaluation of the uncertainties on CMM and on AACMM. This principle has been developed with CETIM for COFRAC accreditation on CMM for gear measurands. The simulation is divided into two principle stages, namely the first is the comprehensive evaluation of possible changes in the geometry of the CMM and the second step, directly related to the measure of the piece, is the evaluation of the analyzed measurand. For AACMM, same principle is realized but the first level is divided into three sub-levels. The division into several levels has many advantages. Indeed, it makes it easier to understand the key sources of uncertainty and thus optimize processes.
\end{abstract}

\section{Introduction}

La méthode de Monte Carlo, appelée aussi Propagation des distributions, a été introduite en métrologie dans les années 1990 [1] et complète le GUM en 2008 sous la forme d'un supplément 1 [2, 3]. Cette méthode est aujourd'hui la méthode reconnue de référence dans la mesure où elle ne fait pas autant d'hypothèses que la méthode analytique et est presque toujours applicable. Néanmoins, son utilisation n'est pas encore parfaitement optimisée dans la mesure où un grand nombre d'utilisateurs réalise une simulation en une seule étape, à l'image du GUM analytique : « une étape pour un modèle unique décrivant le processus ».

L'objectif de nos travaux est d'utiliser cette méthode au plus proche de la physique, c'est-à-dire en réalisant plusieurs niveaux de simulations, afin d'évaluer, maîtriser et optimiser son processus de mesure à chacune des étapes.

Afin de bien comprendre l'intérêt d'un tel découpage de la simulation, deux exemples sont présentés. Le premier exemple concerne l'évaluation des incertitudes sur MMT des mesurandes de l'engrenage cylindrique droit réalisé dans le cadre d'une des accréditations COFRAC du Centre Technique des Industries Mécaniques (CETIM), $[4,5]$. Le second exemple a été réalisé dans le cadre d'un projet de recherche avec le CETIM et ROMER (Groupe HEXAGON METROLOGY) sur la détermination des incertitudes de mesure lors de l'utilisation des bras polyarticulées portables (AACMM) [6]. L'objectif est d'identifier les principales sources d'incertitudes afin de pouvoir techniquement agir sur les paramètres les plus influents en termes d'incertitudes de mesure.

\section{Principe du Monte Carlo à plusieurs niveaux}

\subsection{Principe de la méthode de Monte Carlo}

La méthode de Monte Carlo peut être résumée simplement à l'aide de la Figure 1. Un modèle numérique (programme informatique) est réalisé pour simuler le processus de mesure. A chaque simulation, une valeur possible de chacun des paramètres d'entrée (sources d'incertitudes) est tirée aléatoirement et le mesurande considéré est calculé. En répétant $\mathrm{N}$ fois cette opération, on obtient les variations possibles de ce mesurande, représentées sur la Figure 1 sous la forme d'un histogramme. Il suffit alors de faire une étude statistique pour afficher le résultat de la simulation.

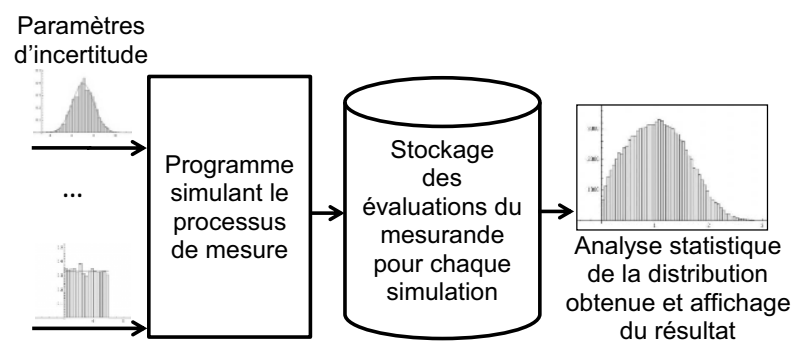

Figure 1. Description de la méthode de Monte Carlo

\subsection{Monte Carlo à plusieurs niveaux}

L'objectif est de réaliser plusieurs simulations de Monte Carlo, correspondant aux grandes étapes du processus de mesure. Dans le cas des machines 3D, nous avons choisi de réaliser $[4,5]$ une simulation à 2 niveaux principaux (Figure 2), à savoir un premier niveau qui permet de

\footnotetext{
${ }^{\mathrm{a}}$ Email de correspondance de l'auteur : francois.hennebelle@u-bourgogne.fr
} 
caractériser les fluctuations possibles de la machine dans ses conditions d'utilisation et un second niveau qui correspond aux fluctuations possibles des différentes pièces mesurées dans l'ensemble des machines possibles. De ce fait, si M simulations sont effectuées au niveau 1 pour caractériser les fluctuations possibles de la machine et que l'on effectue $\mathrm{P}$ simulations pièces dans le second Monte Carlo, alors, au total, pour l'évaluation des incertitudes pour le mesurande considéré, M.P simulations sont effectuées.

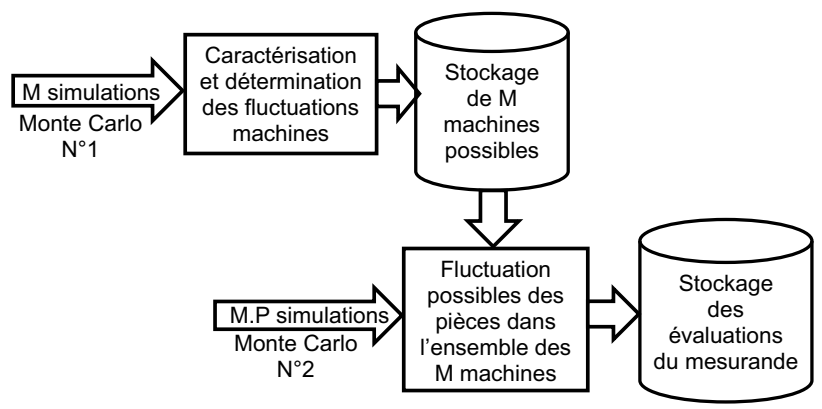

Figure 2. Schéma d'un Monte Carlo à deux niveaux

La réalisation de 2 niveaux présente plusieurs avantages. Tout d'abord, ceci permet de pouvoir gagner du temps de calculs dans les simulations puisque le premier niveau peut dans la majorité des cas être réutilisé pour différentes simulations. De plus, l'étude du premier niveau permet de mettre en place du suivi périodique machine de qualité. En effet, dans la mesure où nous connaissons, par simulation, dans les conditions normales d'utilisation, les fluctuations admissibles de la machine, si l'équipement connait une dérive, il est alors possible très facilement de s'en apercevoir et d'agir en conséquence. Le seul inconvénient de ce découpage en deux niveaux est le nombre de simulations à effectuer qui est plus important. Il convient donc d'optimiser son nombre de tirages $[2,7]$

\section{Applications de la méthode}

Deux exemples d'application sont présentés :

- Evaluation d'un mesurande de division d'un engrenage cylindrique droit sur une MMT

- Evaluation d'incertitudes sur bras polyarticulé portable (AACMM)

\subsection{Cas des Machines à mesurer tridimensionnelles (MMT)}

Ce cas correspond à notre première application de la méthode de Monte Carlo à deux niveaux, qui a été mise en place dans le cadre de l'accréditation COFRAC engrenage sur MMT (Figure 3) au CETIM. L'objectif était de pouvoir évaluer les incertitudes et mettre en place $\mathrm{du}$ suivi périodique de la machine. Dans ce cas, l'utilisation en exploitation de la machine et l'étalonnage sont réalisés dans le même environnement.

La machine utilisé est la MMT du CETIM de Senlis, une Leitz PMM-C 1000P (Figure 3). Pour les mesurandes considérés par cette étude, en ce qui concerne les défauts de la machine, seuls ceux intervenants dans le plan de la mesure sont à déterminer.

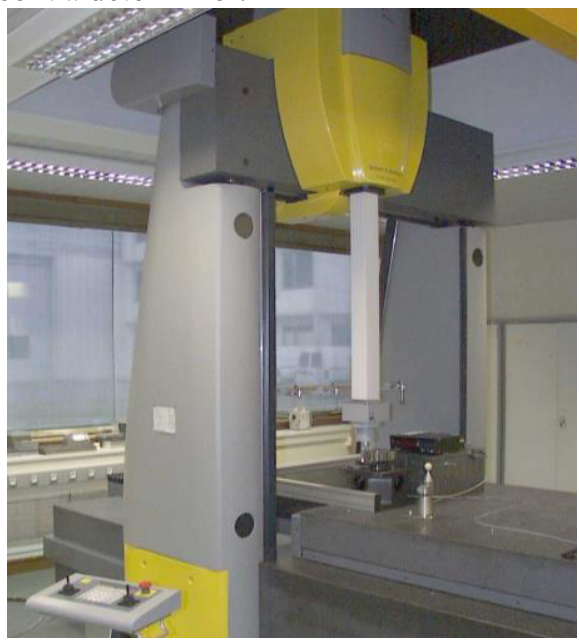

Figure 3. MMT Leitz PMM-C 1000P

Dans le plan, les défauts d'une MMT se résument à deux défauts de justesses, deux de rectitudes, un défaut de lacet et une perpendicularité. Pour déterminer ces défauts, nous avons choisi d'utiliser des étalons physiques, c'est-à-dire une cale à gradins et une règle pour les rectitudes.

\subsubsection{Niveau 1 : fluctuations de la machine}

La simulation Monte Carlo de niveau 1 caractérise alors les fluctuations possibles de ces différents défauts en fonction des principales sources d'incertitudes. Un exemple de fluctuation de la justesse dans l'axe Y de la machine est présenté (Figure 4). Dans [3], les résultats, sur l'ensemble des défauts de la MMT du CETIM, dans le plan sont présentés.

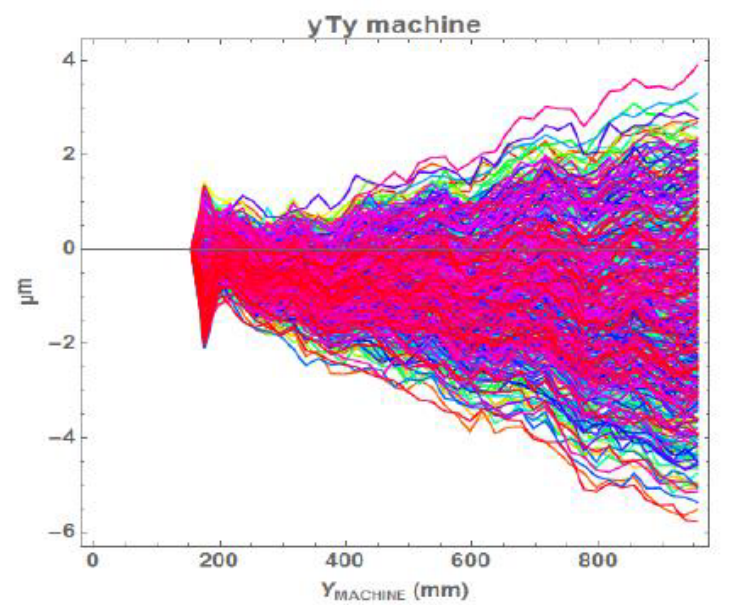

Figure 4. Exemple de variation de la justesse $\mathrm{Y}$ de la MMT

L'analyse des résultats permet de pouvoir mettre en évidence facilement les paramètres influents en termes d'incertitudes et de prendre les décisions ad hoc pour les limiter. Il est alors possible de simuler l'influence de ces défauts sur un étalon typique représentatif de la mesure que l'on cherche à quantifier. On dispose alors d'une référence pour la vérification de la machine. En effet, si la mesure ne se trouve pas dans la bande de tolérance fixé par la simulation, on peut donc en déduire que la 
géométrie de la machine a évolué ou que les conditions extérieures ne sont pas conformes par rapport aux limites fixées. Une roue dentée étant une pièce globalement circulaire, le Machine Checking Gauge (MCG) de RENISHAW, associé à une mesure de cale pour le raccordement au mètre est un bon artefact sur un cercle pour effectuer la vérification périodique. Pour la machine du CETIM, dans les conditions normales d'utilisation, les fluctuations possibles de la mesure de cet artefact est donné par l'ensemble des courbes de la Figure 5. Cet ensemble de courbes possibles constitue alors la « bande de confiance », d'amplitude maximum de 9 micromètres dans le cas présenté. La mesure courante de cet artefact au moment d'une des vérifications, avant une mesure sous accréditation, est également représentée. Dans ce cas, la mesure courante étant dans la bande de confiance, la machine est métrologiquement validée.

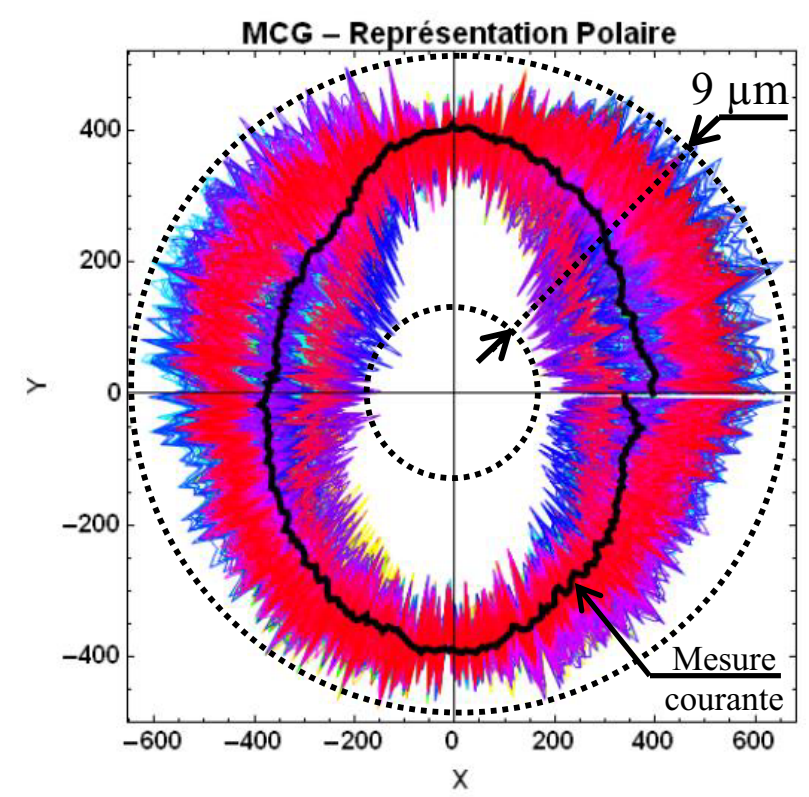

Figure 5. Exemple de suivi périodique avec le MCG

Cette analyse permet également de connaître les directions préférentiels de mesure sur la machine, c'est-àdire celles qui représentent une incertitude plus faible.

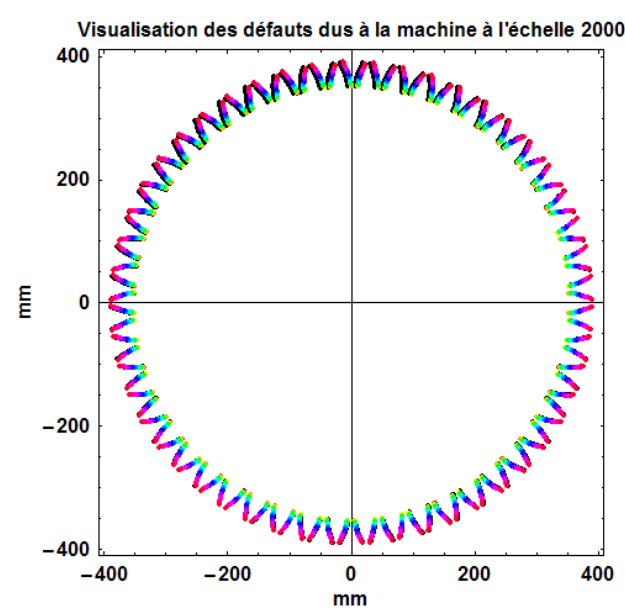

Figure 6. Simulation des défauts de la machine sur la mesure de la roue dentée considérée
Il est également possible de visualiser les incertitudes et la conséquence des défauts résiduels de la machine sur l'ensemble des points théoriques de la pièce réelle mesurée. Si, par exemple, on s'intéresse à la mesure d'une roue dentée de 50 dents $(Z=50)$ de module $m=6$ (Figure 6), les écarts dans la direction de la normale dus à la MMT sur la mesure de la roue (Figure 7) sont identifiables et varient globalement entre $-3 \mu \mathrm{m}$ et $+3 \mu \mathrm{m}$. Ces fluctuations ne peuvent être facilement pris en compte et corrigés si on effectue un Monte Carlo global.

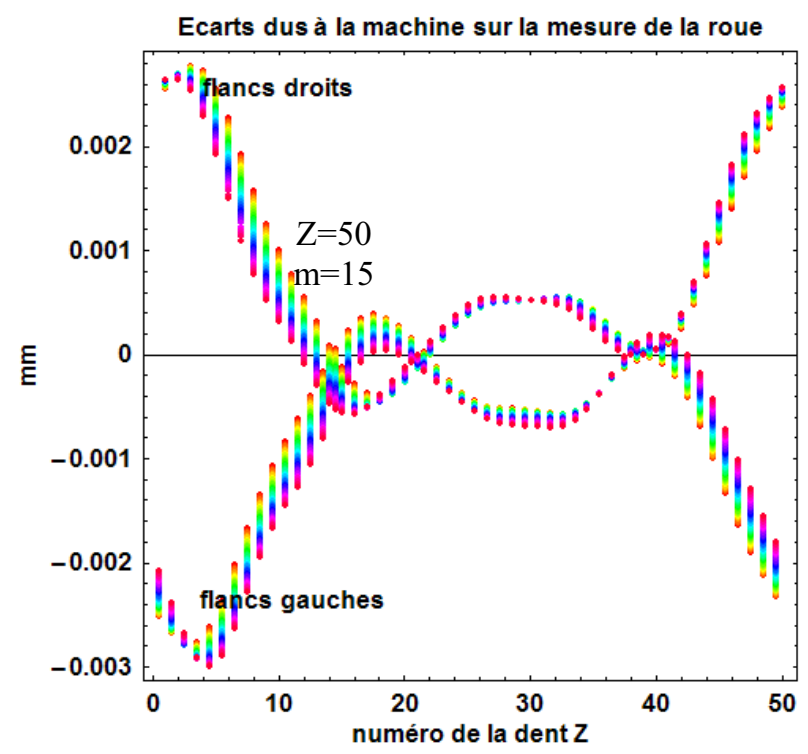

Figure 7. Effet des défauts résiduels de la machine et des incertitudes associées

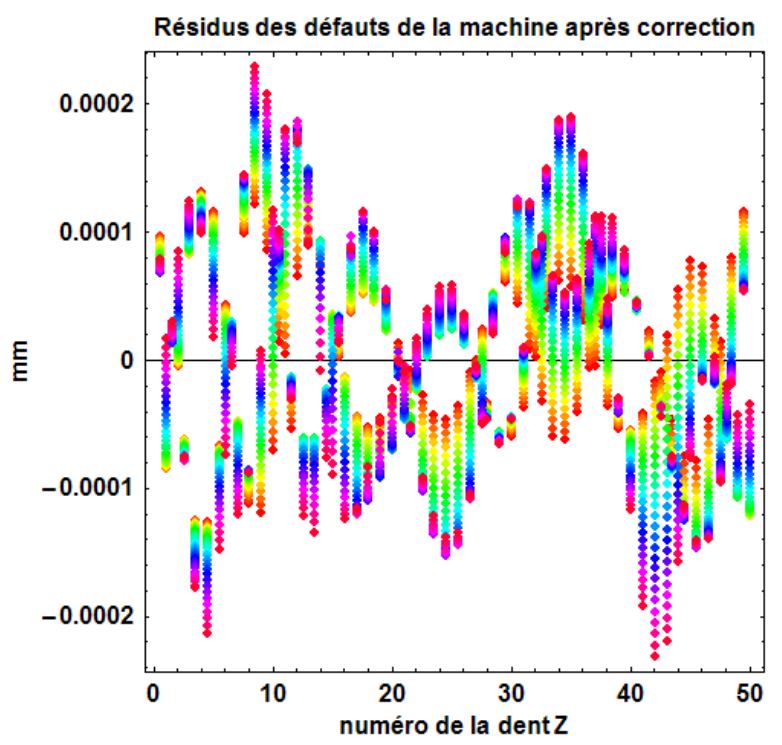

Figure 8. Incertitudes résiduels liées aux fluctuations des défauts de la machine après correction des défauts moyens

Le fait de découper en deux niveaux la simulation permet de pouvoir identifier correctement les défauts de la machine et leurs fluctuations possibles et ainsi de corriger les mesures. Les erreurs résiduelles des défauts de la machine sont alors de quelques dixièmes de micromètres 
(Figure 8), ce qui permet un gain relativement important en termes d'incertitudes de mesure.

\subsubsection{Niveau 2 : fluctuations pièces et évaluation des mesurandes}

Le second niveau consiste à étudier les fluctuations possibles de la pièce parmi l'ensemble des machines simulées et à évaluer pour chaque tirage le ou les mesurandes considérés. Un exemple d'évaluation de mesurande sur la roue dentée présentée précédemment est fourni sur la Figure 9.

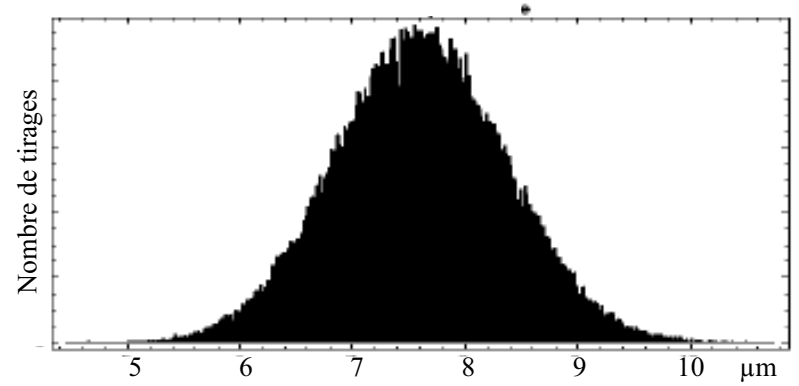

Figure 9. Exemple pour le $\mathrm{f}_{\mathrm{pt}}$ des flancs gauches

Ce niveau 2 permet aussi de pouvoir tester facilement l'influence de la pièce seule sur les incertitudes du mesurande. Il est aussi possible d'optimiser la mesure de celle-ci en analysant les résultats et en travaillant sur les paramètres les plus influents, comme les prises de références et de voir directement l'impact en termes d'incertitudes de mesure...

\subsubsection{Bilan de l'application MMT}

La méthode est éprouvée et validée. Le CETIM utilise ce principe depuis l'accréditation COFRAC des mesurandes des roues dentées sur MMT. L'utilisation du Monte Carlo à deux niveaux a permis de prendre beaucoup de recul sur les sources prépondérantes en termes d'incertitudes et de les limiter un maximum. De plus, les méthodes de suivis mises en place sont alors en adéquation avec le besoin. Le travail permet au CETIM d'être serein lors de ses contrôles en maîtrisant parfaitement son « outil » et son processus de mesure.

\subsection{Cas des bras polyarticulés portables}

Les AACMM (bras polyarticulés portables) sont des machines de mesures, manuelles, non bijectives (plusieurs configurations possibles du bras pour une même mesure), constituées principalement de poutres et d'axes de rotations (Figure 10). Une évaluation d'incertitudes par simulation de Monte Carlo à 2 niveaux a également été mise en place sur différents mesurandes. Le premier niveau a pour objectif de déterminer les incertitudes liées à la machine déterminées en tant que variations des coordonnées du point mesuré. Le second niveau est construit de la même façon que celui pour les MMT et permet d'évaluer les incertitudes sur le mesurande considéré, en faisant intervenir les fluctuations possibles de la pièce et de la mesure de celleci liées aux prises de référence, bridage, variations de température... Ce second niveau ne sera pas présenté.

Le premier niveau est découpé en trois sous niveaux (Figure 12) afin de pouvoir comprendre physiquement l'origine des incertitudes de mesure et de mieux appréhender la machine.

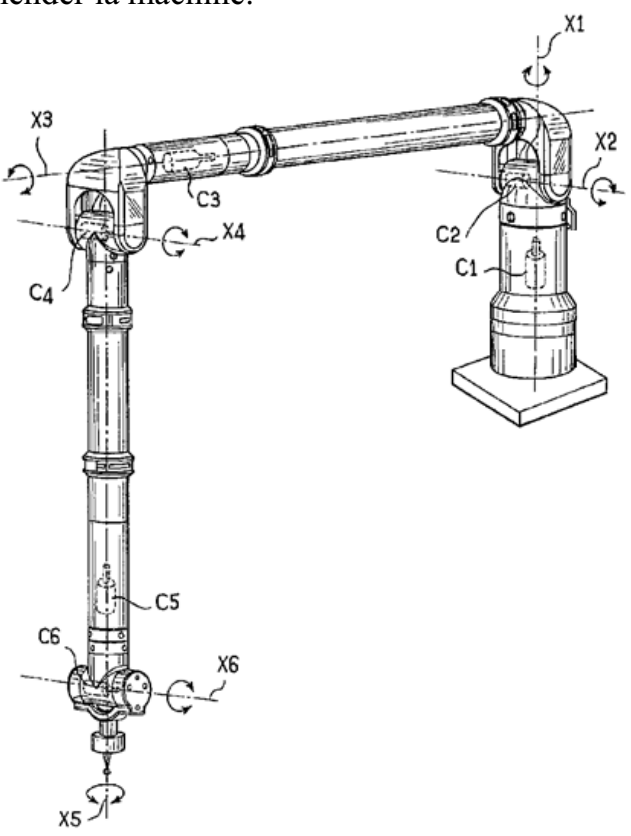

Figure 10. Schéma de principe d'un AACMM

\subsubsection{Niveau 1.1}

Le niveau 1.1 correspond globalement à la quantification des variations possibles des coordonnées cartésiennes lors de la mesure d'un point et permet alors de simuler la répétabilité sur point effectué en utilisant l'étalon à 3 billes (Figure 11) et le capteur de référence. Les erreurs liées à l'étalonnage n'interviennent pas dans ce cas.

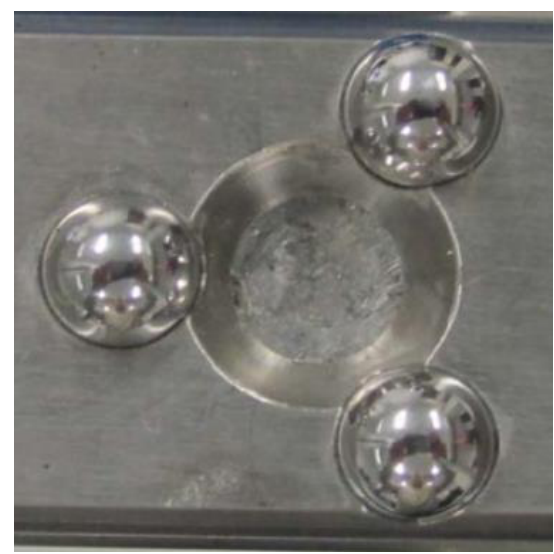

Figure 11. Etalon à 3 sphères pour l'évaluation de la répétabilité de mesure d'un point

Ce niveau est très important dans le cadre de l'étude du dispositif pour un fabricant de matériel. En effet, il permet de mettre en place un modèle de l'AACMM afin de comprendre et d'identifier les principales sources d'incertitudes lors des mesures de points. Ce niveau et les principales sources d'incertitudes sont décrits dans [6]. 


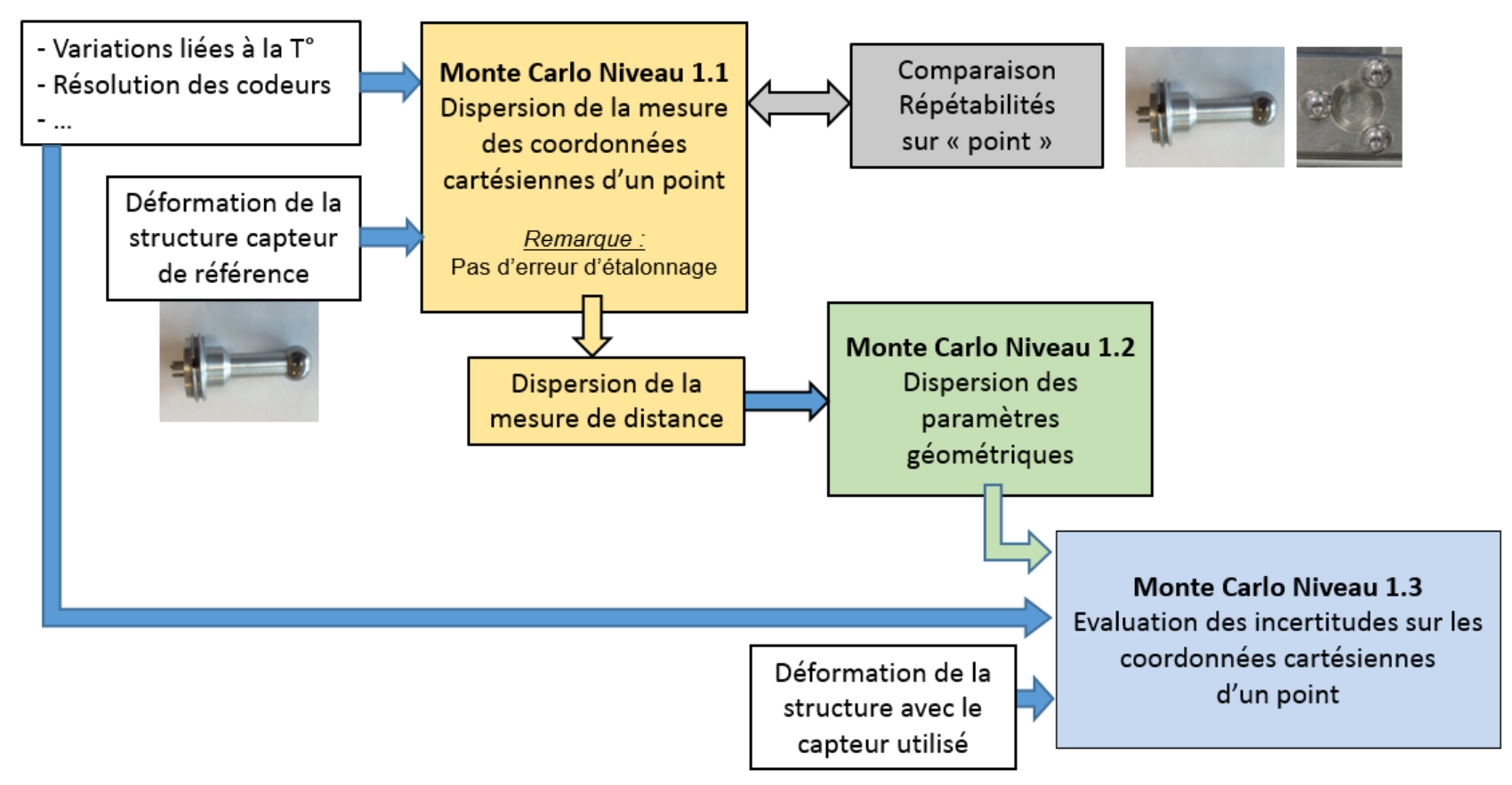

Figure 12. Schéma du Monte Carlo de Niveau 1 pour un AACMM

Pour un utilisateur, le résultat d'une répétabilité sur point permet globalement de réaliser ce niveau en intégrant les incertitudes liées aux vibrations et aux déformations de la structure par l'opérateur.

\subsubsection{Niveau 1.2}

Le niveau 1.2 correspond à la détermination des incertitudes sur les paramètres d'étalonnage de l'AACMM, c'est-à-dire les erreurs sur la détermination des longueurs des différentes poutres et de la position initiale des codeurs angulaires. Classiquement, cet étalonnage est réalisé par le fabricant en usine. Pour effectuer cet étalonnage, des distances sont mesurées dans différentes direction dans l'espace de travail selon la norme ASME B89.4.22-2004 [8]. Les longueurs et angles sont alors optimisés par méthode inverse. Cette étude permet d'évaluer les erreurs de justesse résiduels du bras de mesure, qui correspondent à des erreurs d'étalonnage. En effet, la détermination des paramètres d'étalonnage est moyenne sur l'ensemble des positions mesurés. Ce niveau est très important pour un fabricant afin de chiffrer les incertitudes sur les paramètres d'étalonnage et optimiser les positions et mesures lors de la phase d'étalonnage mais aussi pour connaitre les zones de mesure optimales.

Ce niveau peut difficilement être effectué par un utilisateur dans la mesure où celui-ci ne connaît pas le protocole d'étalonnage de l'AACMM réalisé en usine. Néanmoins l'utilisateur peut estimer les erreurs résiduels d'étalonnage en mesurant une cale étalon dans les différentes configurations utilisées lors de la mesure de sa pièce. A titre d'exemple, si on considère que pour mesurer une pièce donnée, l'AACMM est utilisé en position verticale $(\mathrm{V})$ ou horizontal $(\mathrm{H})$ et qu'en fonction de l'accès permis, la face est accostée par le palpeur tel que l'axe du stylet est soit normale $(\mathrm{N})$, soit inclinée (I), soit orthogonal à la surface $(\mathrm{O})$. Une face de cale peut donc être mesurée de 6 façons: VN, VO, VI, HN, HO, HI. Il est alors possible de caractériser les erreurs liées à l'étalonnage lors de la mesure de la longueur de la cale. La Figure 13 présente les résultats pour six configurations possibles de l'évaluation de la longueur de la cale étalon. La première face est toujours mesurée avec le bras en position verticale et la face est accostée avec l'axe du sylet normale à la face (configuration VN que l'on considère comme référence ici) et la seconde est mesurée dans les six configurations possibles (VN, VO, VI, HN, $\mathrm{HO}, \mathrm{HI})$. Avec l'AACMM utilisé, les erreurs résiduelles d'étalonnage varient alors entre -17.5 et $21.4 \mu \mathrm{m}$ en moyenne (Figure 13) ce qui est loin d'être négligeable sur le bras testé. Ce test simple peut servir aussi de suivi périodique de l'équipement.

Distance entre faces d'une cale de $300 \mathrm{~mm}$

5 mesures par configuration et moyenne

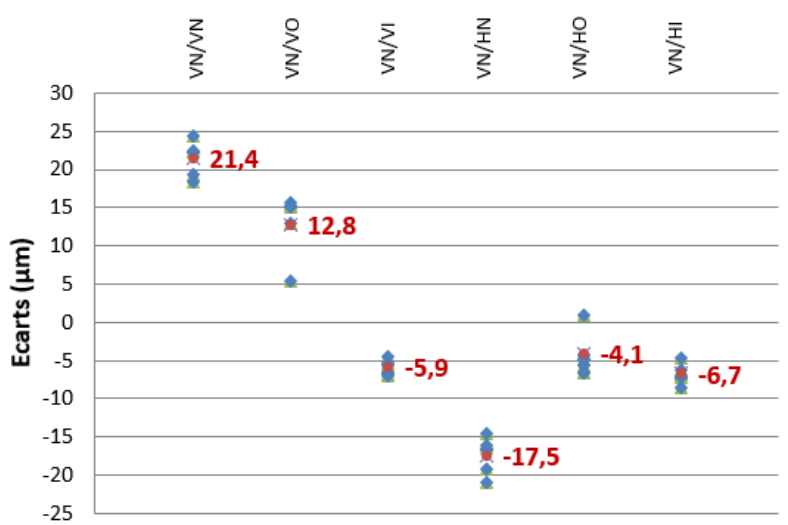

Figure 13. Evaluation d'erreurs résiduelles d'étalonnage d'un bras pour 6 configurations de mesure différentes 


\subsubsection{Niveau 1.3}

Le niveau 1.3 correspond aux incertitudes globales de la machine qui regroupent :

- les paramètres du modèle de niveau 1.1 ou les résultats de la répétabilité sur point pour un utilisateur,

- les incertitudes sur les paramètres d'étalonnage du niveau 1.2 ou l'estimation des erreurs résiduels d'étalonnage estimé expérimentalement pour un utilisateur

- les paramètres du modèle propres à la mesure (choix du capteur...). Ce niveau fourni donc les variations possibles des coordonnées des points théoriques mesurés.

\subsubsection{Bilan sur $A A C M M$}

Nous avons travaillé sur un AACMM ROMER Groupe HEXAGON METROLOGY, de type Sigma 2025 et avons effectué un modèle complet de celui-ci.

Pour le niveau 1.1, les incertitudes-type obtenues sont d'environ $18 \mu \mathrm{m}$, ce qui est cohérent avec la répétabilité sur point.

$\mathrm{Au}$ niveau 1.2, avec une procédure de type ASME B89.4.22-2004, les erreurs liées aux paramètres d'étalonnage correspondent à environ $2 \mu \mathrm{m}$ d'erreurs sur les distances des poutres et de $0.6 \mu \mathrm{rad}$ sur les erreurs de positions des codeurs pour notre modèle.

Au niveau 1.3, l'incertitude-type obtenue est de $58 \mu \mathrm{m}$ sur les coordonnées cartésiennes d'un point mesuré.

Comme précisé au $\$ 3.2$, nous ne présenterons pas de résultats de la simulation de niveau 2 qui permet d'obtenir les incertitudes sur un mesurande donné dans la mesure où cette étape a déjà été évoquée sur l'application MMT.

\section{Conclusion}

Le découpage de la simulation de Monte Carlo en plusieurs niveaux permet de réaliser des évaluations au plus proche de la physique en respectant les différentes étapes qui conditionnent la mesure. Cette démarche est fondamentale lors d'optimisation de processus de mesures. En effet, l'objectif est de comprendre le processus de mesure pour identifier les sources prépondérantes en termes d'incertitudes de mesure afin de pouvoir travailler sur ces paramètres et ainsi améliorer la qualité des mesures.

L'application $\mathrm{n}^{\circ} 1$ sur MMT nous a permis de montrer qu'en détaillant son processus, il était possible de faire des corrections réduisant grandement les incertitudes de mesure (Figure 7 et Figure 8), de mettre en place du suivi périodique optimisé (Figure 5). Cette démarche a permis au CETIM d'obtenir la première accréditation en France sur MMT pour des mesurandes d'engrenages mais aussi d'avoir une expertise importante et un recul sur les mesures effectuées au laboratoire.

L'application $n^{\circ} 2$ permet de montrer qu'il est possible de simuler le processus de mesure complet d'un AACMM en évaluant les différentes étapes qui conduisent à la mesure. Cette étude est fondamentale pour un fabricant qui cherche à optimiser ses produits. En effet, la simulation lui permet d'identifier rapidement les sources de progrès sur ses produits tel que le choix des matériaux, l'optimisation des positions d'étalonnage, évaluation de nouvelles solutions techniques....

Pour un utilisateur, ceci lui permet de savoir chiffrer ses incertitudes de mesure, mais aussi de mieux comprendre la physique de la mesure et donc optimiser ses protocoles de mesure.

Il est possible de mettre en place également ce type de méthode sur ligne afin d'optimiser la production. Faut-il produire plus et plus vite ou produire optimisé, c'est-àdire mieux maîtriser les variabilités et donc consommer juste ce qu'il faut?

\section{Références}

1. T. Coorevits, J.M. David, Incertitudes de mesure des machines à mesurer tridimensionnelle: approche par une méthode de Monte-Carlo, Bulletin du Bureau National de Métrologie No97, (1994)

2. JCGM 101:2008, Evaluation of measurement data Supplement 1 to the "Guide to the expression of uncertainty in measurement" - Propagation of distributions using a Monte Carlo method, (2008).

3. F. Hennebelle, T. Coorevits, (2013), Propagation des distributions - Détermination des incertitudes par la méthode de Monte Carlo, Techniques de l'Ingénieur, r288

4. T. Coorevits, F. Hennebelle, P. Sessa, N. Rousset, Démarche d'accréditation en métrologie des engrenages pour les mesurandes normalizes sur machine à mesurer tridimensionnelle, $14^{\text {ème }}$ Congrès International de Métrologie, (22 au 25 juin 2009).

5. F. Hennebelle, T. Coorevits, P. Sessa, Présentation de la première accreditation COFRAC sur machine à mesurer tridimensionnelle, $15^{\text {ème }}$ Congrès International de Métrologie, (2 au 6 octobre 2011).

6. F. Romdhani, F. Hennebelle, M. Ge, P. Juillion, R. Coquet, J.-F. Fontaine, Methodology for the assessment of measuring uncertainties of articulated arm coordinate measuring machines ,Measurement Science \& Technology, (2014), 25 (125008), 14pp

7. Wübbeler et al. (2010), Metrologia 47317

8. ASME B89.4.22-2004: Methods for Performance Evaluation of Articulated Arm Coordinate Measuring Machines. (2005, August 12). U.S.A: The American Society of Mechanical Engineers. 